\title{
Geographical Variability in Paromomycin Pharmacokinetics Does Not Explain Efficacy Differences between Eastern African and Indian Visceral Leishmaniasis Patients
}

\author{
Luka Verrest $^{1} \cdot$ Monique Wasunna $^{2} \cdot$ Gilbert Kokwaro $^{3,4} \cdot$ Rashid Aman $^{4} \cdot$ Ahmed M. Musa $^{5}$ - Eltahir A. G. Khalii ${ }^{5}$. \\ Mahmoud Mudawi ${ }^{5,6}$. Brima M. Younis ${ }^{5}$. Asrat Hailu ${ }^{7}$. Zewdu Hurissa ${ }^{8}$. Workagegnehu Hailu ${ }^{9}$. Samson Tesfaye ${ }^{7}$. \\ Eyasu Makonnen ${ }^{7}$. Yalemtsehay Mekonnen ${ }^{10}$. Alwin D. R. Huitema ${ }^{1,11,12}$. Jos H. Beijnen ${ }^{1}$ - Smita A. Kshirsagar ${ }^{13}$. \\ Jaya Chakravarty ${ }^{14} \cdot$ Madhukar Rai $^{14}$. Shyam Sundar ${ }^{14}$. Fabiana Alves ${ }^{15} \cdot$ Thomas P. C. Dorlo $^{1}$
}

Accepted: 4 May 2021 / Published online: 9 June 2021

(c) The Author(s) 2021, corrected publication 2022

\begin{abstract}
Introduction Intramuscular paromomycin monotherapy to treat visceral leishmaniasis (VL) has been shown to be effective for Indian patients, while a similar regimen resulted in lower efficacy in Eastern Africa, which could be related to differences in paromomycin pharmacokinetics.

Methods Pharmacokinetic data were available from two randomized controlled trials in VL patients from Eastern Africa and India. African patients received intramuscular paromomycin monotherapy ( $20 \mathrm{mg} / \mathrm{kg}$ for 21 days) or combination therapy $(15 \mathrm{mg} / \mathrm{kg}$ for 17 days) with sodium stibogluconate. Indian patients received paromomycin monotherapy (15 $\mathrm{mg} / \mathrm{kg}$ for 21 days). A population pharmacokinetic model was developed for paromomycin in Eastern African and Indian VL patients. Results Seventy-four African patients (388 observations) and 528 Indian patients (1321 observations) were included in this pharmacokinetic analysis. A one-compartment model with first-order kinetics of absorption and elimination best described paromomycin in plasma. Bioavailability (relative standard error) was 1.17 (5.18\%) times higher in Kenyan and Sudanese patients, and $2.46(24.5 \%)$ times higher in Ethiopian patients, compared with Indian patients. Ethiopian patients had an approximately fourfold slower absorption rate constant of $0.446 \mathrm{~h}^{-1}(18.2 \%)$. Area under the plasma concentration-time curve for $24 \mathrm{~h}$ at steady-state $\left(\mathrm{AUC}_{\tau, \mathrm{SS}}\right)$ for $15 \mathrm{mg} / \mathrm{kg} /$ day (median [interquartile range]) was higher in Kenya and Sudan $(172.7$ $\mu \mathrm{g} \cdot \mathrm{h} / \mathrm{mL}$ [145.9-214.3]) and Ethiopia (230.1 $\mu \mathrm{g} \cdot \mathrm{h} / \mathrm{mL}$ [146.3-591.2]) compared with India (97.26 $\mu \mathrm{g} \cdot \mathrm{h} / \mathrm{mL}$ [80.83-123.4]). Conclusion The developed model provides detailed insight into the pharmacokinetic differences among Eastern African countries and India, however the resulting differences in paromomycin exposure do not seem to explain the geographical differences in paromomycin efficacy in the treatment of VL patients.
\end{abstract}

\section{Introduction}

Over the past decades, substantial progress has been made in the treatment of visceral leishmaniasis (VL). Geographical differences in treatment efficacy have led to variable treatment recommendations for VL, with intramuscular paromomycin being one of the recommended treatment options in regions where VL is endemic. In South Asia, a 10-day

\footnotetext{
Luka Verrest

1.verrest@nki.nl

Thomas P. C. Dorlo t.dorlo@nki.nl
}

Extended author information available on the last page of the article combination of paromomycin and miltefosine is recommended as second-line treatment, while in Eastern Africa, a 17-day combination of paromomycin and sodium stibogluconate (SSG) is recommended by the World Health Organization (WHO) [1].

While paromomycin is affordable and has a reasonable safety profile compared with the conventional treatment of VL with antimonial compounds, efficacy of paromomycin monotherapy has been shown to be highly variable between geographical regions where VL is endemic. Intramuscular paromomycin sulfate ('paromomycin') as monotherapy at a dose of $15 \mathrm{mg} / \mathrm{kg}$ for 21 days was highly effective in Indian VL patients (final cure rate 94.6\%), assessed 6 months after the end of treatment [2], but failed to show a similar efficacy 


\section{Key Points}

Intramuscular paromomycin is one of the recommended treatment options in regions where visceral leishmaniasis (VL) is endemic, although efficacy has been shown to be highly variable between geographical regions.

To explore if the geographical differences in efficacy might be caused by variability in pharmacokinetics, the pharmacokinetics of paromomycin were investigated in Eastern African and Indian patients.

Although paromomycin pharmacokinetics can most probably not explain the efficacy differences, remarkable differences in exposure were observed between VL patient populations, demonstrating the relevance of pharmacokinetic analysis in VL clinical trials globally.

in Eastern Africa. Within Eastern Africa, large geographical variability in efficacy of this treatment regimen has been observed, with lowest efficacy in sites in Sudan (14.3\% and 46.7\%) compared with Kenya (80.0\%), Northern Ethiopia (75.0\%), and Southern Ethiopia (96.6\%) [3]. A dose increase $(20 \mathrm{mg} / \mathrm{kg}$ for 21 days) or longer treatment duration $(15 \mathrm{mg} /$ $\mathrm{kg}$ for 28 days) resulted in an improved but still insufficient paromomycin monotherapy efficacy in Sudan of $80 \%$ and $81 \%$, respectively [4]. A combination therapy of $15 \mathrm{mg} / \mathrm{kg}$ paromomycin for 17 days plus SSG showed an adequate efficacy of $91.4 \%$ in Eastern Africa [5].

As variability in drug exposure might explain the geographical and regional differences in the clinical efficacy of paromomycin monotherapy between India and Eastern Africa, but also within Eastern Africa, paromomycin pharmacokinetics should be investigated. In general, pharmacokinetic studies to optimize the treatment of VL are lacking $[6,7]$. Likewise, little is known about the pharmacokinetics of paromomycin after intramuscular injection; a pharmacokinetic study in 15 healthy volunteers has been conducted [8], two clinical studies reported paromomycin plasma concentrations in 9 Sudanese [4] and 453 Indian VL patients [2]. Paromomycin is poorly absorbed after oral administration [9, 10], however after intramuscular administration, absorption is fast, with peak concentrations typically between 1 and $2 \mathrm{~h}$ after administration $[2,4,8]$, and bioavailability is expected to be nearly $100 \%$ [10]. Paromomycin is a hydrophilic compound with moderate plasma protein binding capacity of 33\% [9] and limited distribution to tissues (approximately $40 \%$ of body weight) $[8,10]$, and is mainly excreted unchanged by glomerular filtration $[9,10]$. However, only $60.1 \%$ urine recovery has been reported after a $15 \mathrm{mg} / \mathrm{kg}$ paromomycin dose [8], suggesting that absorption might not be complete. Elimination of paromomycin is characterized by a short half-life of $2-3 \mathrm{~h}$ and no dose accumulation has been observed with daily paromomycin dosing [8-10]. Whether there is variability in exposure between VL patients from different geographical regions following intramuscular paromomycin therapy is currently unknown.

To explore if the geographical differences in efficacy might be caused by variability in pharmacokinetics, the pharmacokinetics of paromomycin among Eastern African countries and India was investigated. To allow for analysis of sparse and heterogeneous data, a population approach was applied for pharmacokinetic modelling of paromomycin in Eastern African and Indian VL patients. A covariate analysis was performed to identify variables explaining the heterogeneity, including demographic differences and patient characteristics reflecting the patient's health status and renal function.

\section{Methods}

\subsection{Study Design and Patients}

Pharmacokinetic data were available from a subset of patients from two open-label, multicentre, randomized controlled trials in VL patients in Eastern Africa [5] (NCT00255567) and India [2] (NCT00216346). Eastern African patients were enrolled at four clinical trial sites in Kassab, Sudan; Gondar, Northern Ethiopia; Arba Minch, Southern Ethiopia; and Nairobi, Kenya, and Indian patients were enrolled at four clinical trial sites in Bihar, India. Patients aged 4-60 years (Africa) or 5-55 years (India) with parasitologically confirmed VL were included, but patients with severe VL or comorbidities, including HIV co-infection, were excluded from the trials. In addition, patients weighing $<30 \mathrm{~kg}$ (Kenya and Sudan) or who were $<7$ years of age (Ethiopia) were excluded from the pharmacokinetic substudy. Other inclusion and exclusion criteria have been described previously [2, 3].

\subsection{Study Medication}

Paromomycin solution, containing $375 \mathrm{mg}$ paromomycin base (500 mg paromomycin sulfate) per millilitre, produced at Pharmamed Parenterals Ltd (PPL), Malta (Indian trial) or Gland Pharma, India (African trial), was administered intramuscularly into the gluteus muscle. A salt correction factor of 0.7554 (India) or 0.733 (Africa) was used to convert the paromomycin sulfate dose to paromomycin base. Indian patients received paromomycin monotherapy (15 $\mathrm{mg} / \mathrm{kg}$ paromomycin sulfate for 21 days), while African patients received paromomycin monotherapy $(20 \mathrm{mg} / \mathrm{kg}$ paromomycin sulfate for 21 days) or paromomycin plus SSG 
combination therapy $(15 \mathrm{mg} / \mathrm{kg}$ paromomycin sulfate and 20 $\mathrm{mg} / \mathrm{kg}$ SSG for 17 days).

\subsection{Sample Collection}

Blood samples were drawn from patients in Sudan and Kenya according to an intensive schedule, with samples drawn before treatment administration and 0.5, 1, 3, 6, 10, and $24 \mathrm{~h}$ after treatment administration on the first and last day of treatment (day 21 in the monotherapy arm, day 17 in the combination arm). In Ethiopia, blood samples (three per patient) were drawn according to a sparse schedule on days 7, 14, or 21 for the monotherapy arm and on days 7, 14 , or 17 for the combination arm. In India, blood samples (three per patient) were collected at prespecified times over the course of the 21-day study for the purposes of population modelling.

\subsection{Bioanalysis}

Plasma samples were analysed by high-performance liquid chromatography coupled to UV detection (HPLC-UV) upon pre-column derivatization of paromomycin (Africa) or by liquid chromatography tandem mass spectrometry (LC/MS/ MS) [India]. A description of the assay and assay validation can be found in electronic supplementary material (ESM) 1 .

\subsection{Population Pharmacokinetic Analysis}

To allow for analysis of sparse and heterogeneous data, a population approach was applied for pharmacokinetic modelling of paromomycin plasma concentrations. The population pharmacokinetic model was developed using a nonlinear mixed-effects modelling approach (NONMEM, version 7.3; ICON Development Solutions, Ellicott City, MD, USA) using the first-order conditional estimation method with interaction (FOCE-I) and ADVAN13. Tools used to evaluate the model and visualize the data and model output were $\mathrm{R}$ (version 3.6.2), RStudio (version 1.2.5033; Boston, MA, USA), PsN (version 4.7.0), and the graphical interface Pirana (version 2.9.9). Model development and evaluation was performed in three steps: (1) selection of a structural and stochastic model; (2) selection of the covariate model; (3) internal model evaluation.

\subsection{Structural and Stochastic Model Development}

During development of the structural pharmacokinetic model, one- and two-compartment models with first-order absorption and elimination were tested to fit paromomycin plasma concentration-time data. Different methods for handling below the limit of quantification (BLQ) were investigated: BLQ observations set to half the limit of quantitation, the M3 method, or excluding BLQ observations [11]. In the stochastic model, between-subject variability was modelled assuming a log-normal distribution (Eq. 1), while residual variability was modelled using a proportional error model (Eq. 2).

$P_{i}=P_{\text {pop }} \times e^{\eta_{i}}$

$Y_{i, j}=C_{i, j} \times(1+\varepsilon)$.

where $P_{\text {pop }}$ is the population estimate for a parameter, $P_{i}$ is the individual (post hoc) value for that parameter, and $\eta_{i}$ is the between-subject variability of the $i$ th individual with mean zero and variance $\omega^{2} . Y_{i, j}$ is the observed concentration and $C_{i, j}$ is the predicted concentration for the $j$ th observation of the $i$ th individual, and $\varepsilon$ is the residual error with mean zero and variance $\sigma^{2}$. A separate residual variability was estimated for the two studies, as pharmacokinetic samples were processed and analysed differently.

\subsection{Covariate Model Development}

In the covariate model, time-varying body weight was included a priori, with fixed allometric exponents of 0.75 for clearance and 1.00 for volume of distribution [12]. Other covariates were tested on absorption rate constant, bioavailability, volume of distribution, and clearance. SSG comedication was evaluated as a binary covariate to check for potential drug-drug interactions. Paromomycin dose level ( $15 \mathrm{vs.} 20 \mathrm{mg} / \mathrm{kg} /$ day) was evaluated as a binary covariate to check for dose proportionality. Evaluated patient characteristics included baseline age, and time-varying serum creatinine, standardized glomerular filtration rate (GFR) for a typical body surface area (BSA) of $1.73 \mathrm{~m}^{2}$, absolute GFR unadjusted to typical BSA $\left(\mathrm{GRF}_{\mathrm{abs}}\right)$, and serum albumin. GFR was calculated according to the Modification of Diet in Renal Disease (MDRD) formula [13] (Eq. 3).

$$
\begin{aligned}
\text { GFR }= & 32788 \times \text { serum creatinine } e^{-1.154} \\
& \times \text { age }^{-0.203} \times(0.742 \text { if female }),
\end{aligned}
$$

where GFR is expressed in millilitres per minute per $1.73 \mathrm{~m}^{2}$ $\left(\mathrm{mL} / \mathrm{min} / 1.73 \mathrm{~m}^{2}\right)$, age is expressed in years, and serum creatinine is expressed in micromoles per litre $(\mu \mathrm{mol} / \mathrm{L})$. $\mathrm{GFR}_{\mathrm{abs}}$ was calculated by multiplying the original GFR with the individual BSA [14] (Eqs. 4 and 5).

$\mathrm{GFR}_{\mathrm{abs}}=\frac{\mathrm{GFR}}{1.73} \times \mathrm{BSA}$

BSA $=0.20247 \times$ height $^{0.725} \times$ weight $^{0.425}$,

where BSA is expressed in metres squared $\left(\mathrm{m}^{2}\right)$, height is expressed in metres $(\mathrm{m})$, and weight is expressed in 
kilograms $(\mathrm{kg})$. Serum creatinine and GFR may reflect renal function, which may impact clearance of the renally cleared drug paromomycin. VL patients are haematologically depleted [15] and the majority of patients are malnourished. Serum albumin, which is usually decreased in VL patients, may be influenced by both processes and may represent the overall health status of the patient, possibly impacting paromomycin absorption, distribution, or clearance. Furthermore, time after the start of treatment was tested as a covariate to assess whether pharmacokinetic parameters changed during treatment, e.g. due to overall clinical improvement of the patient. To investigate geographical differences that were not explained by plausible physiological covariates, country and study sites were evaluated as covariates, e.g. representing other unaccounted demographical differences or study-specific procedures. Missing baseline covariates were handled by implementing the median value in the study population, or, in case of time-varying covariates, using the last observation measured in that subject carried forward.

To assess covariate relations, post hoc values and between-subject variability were plotted against covariates. Continuous covariates were tested in a linear, exponential, and power function (Eqs. 6-8):

$$
\begin{aligned}
& P_{\mathrm{TV}}=P_{\mathrm{pop}} \times\left(1+\left(\operatorname{Cov}_{i, t}-\operatorname{Cov}_{\mathrm{med}}\right)\right) \times l \\
& P_{\mathrm{TV}}=P_{\mathrm{pop}} \times e^{\left(\operatorname{Cov}_{i, t}-\operatorname{Cov}_{\mathrm{med}}\right) \times h} \\
& P_{\mathrm{TV}}=P_{\mathrm{pop}} \times\left(\frac{\operatorname{Cov}_{i, t}}{\operatorname{Cov}_{\mathrm{med}}}\right)^{k}
\end{aligned}
$$

where $P_{\text {pop }}$ represents the population estimate of this parameter, $P_{\mathrm{TV}}$ is the typical parameter value at covariate value $\operatorname{Cov}_{i, t}, \operatorname{Cov}_{i, t}$ is the covariate value for the $i$ th individual at time $t$, and $\operatorname{Cov}_{\text {med }}$ is the median covariate value in the population. $\mathrm{Cov}_{\text {med }}$ was set to 0 for time. In the linear function (Eq. 6), $l$ represents the slope factor, and $h$ and $k$ represent the scaling factors in the exponential (Eq. 7) and power function (Eq. 8). Clearance was also evaluated as a fraction of $\mathrm{GFR}_{\mathrm{abs}}$, where renal clearance is assumed to be the only route of elimination. Categorical covariates were tested as proportional changes relative to the reference category.

\subsection{Model Selection and Evaluation}

Model selection was based on scientific plausibility, minimum objective function value (OFV), goodness-of-fit $(\mathrm{GoF})$ plots, and precision of parameter estimates. A decrease in OFV over 6.63 points between nested models was considered statistically significant, corresponding to a $p$-value of $<0.01$ following a Chi-square distribution with 1 degree of freedom. The final population pharmacokinetic model was evaluated by GoF plots [16], a prediction-corrected visual predictive check (VPC) [17], and sampling-importance resampling (SIR) [18]. SIR was used to derive the 95\% confidence interval $(\mathrm{CI})$ of the model parameters. Paromomycin exposure for the different countries was derived using the final individual pharmacokinetic model estimates, expressed by the area under the plasma concentration-time curve for $24 \mathrm{~h}$ at steady-state $\left(\mathrm{AUC}_{\tau, \mathrm{SS}}\right)$ determined on the last day concentrations were measured.

\section{Results}

\subsection{Patients and Data}

Data from 74 African patients (388 observations) and 448 Indian patients (933 observations) were used for the pharmacokinetic analysis (Fig. 1; Fig. S1B in ESM 2; Table 1 and Table 2). Reliable serum creatinine levels were only available for patients from Kenya and Ethiopia, with baseline creatinine levels almost all within the normal range of 50-130 $\mu \mathrm{mol} / \mathrm{L}$ (observed range 44.2-134 $\mu \mathrm{mol} / \mathrm{L}$ ) (Table 1). As expected for VL patients, baseline albumin levels, which were available for all Kenyan, Ethiopian and Sudanese patients, were low (observed range 7-40 $\mathrm{g} / \mathrm{L})$ (Table 1), but levels increased during treatment and follow-up (Fig. S2 in ESM 2). Six observations from three subjects were excluded from this analysis because paromomycin concentrations were extremely high $(n=3)$ or sampling time was missing $(n=3)$. In both the African and Indian data, observations $24 \mathrm{~h}$ after the last administered dose, right before the next dose, were highly variable and in some cases physiologically implausible, whereas observations at the time of dosing were always BLQ (Fig. S1A in ESM 2). Therefore, it was likely that these samples were collected after dosing and therefore all observations around the time of dosing (time after dose $[\mathrm{TAD}]=0$ or $24 \mathrm{~h}$ ) were excluded from the analysis. In the final dataset, only 9 (2.3\%) African and 34 (3.5\%) Indian BLQ observations were present. Exclusion of BLQ observations did not lead to changes in model fit, and therefore these observations were excluded from the pharmacokinetic analysis.

\subsection{Population Pharmacokinetic Model}

A one-compartment model with first-order kinetics of absorption and elimination described the available paromomycin observations in plasma adequately. The typical values (relative standard error [RSE]) for clearance and volume of distribution of paromomycin were estimated at $4.38 \mathrm{~L} / \mathrm{h}$ (2.36\%) and $15.6 \mathrm{~L}$ (2.02\%), respectively (Table 3). Between-subject variability (CV\% [RSE\%]) could be identified for clearance $(33.2 \%$ [10.4\%]) for all subjects, and, 
Table 1 Patient characteristics

\begin{tabular}{|c|c|c|c|c|c|}
\hline & Kenya & Sudan & Ethiopia & India & Total \\
\hline Subjects $(n)$ & 16 & 16 & 42 & 454 & 528 \\
\hline Male $[n(\%)]$ & $13(81)$ & $13(81)$ & $34(81)$ & $296(65)$ & $356(67)$ \\
\hline Age, years [mean (range)] & $23.1(15-45)$ & $25.4(12-40)$ & $19.9(8-60)$ & $22.5(5-54)$ & $22.4(5-60)$ \\
\hline Paediatric patients $\mathrm{s}^{\mathrm{a}}[n$ (\% of total $\left.)\right]$ & $3(19)$ & $3(19)$ & $18(43)$ & $190(42)$ & $214(41)$ \\
\hline Body weight, kg [mean (range)] ${ }^{\mathrm{b}}$ & $47.3(37-56)$ & $49.1(34-73)$ & $39.4(15-62)$ & $35.8(11-68)$ & $36.8(11-73)$ \\
\hline Creatinine, $\mu \mathrm{mol} / \mathrm{L}[\text { mean (range) }]^{\mathrm{b}}$ & $95.3(79.6-134)$ & NA & $79.5(44.2-115)$ & NA & $83.8(44.2-134)$ \\
\hline eGFR, mL/min/1.73 $\mathrm{m}^{2}$ [mean (range) $]^{\mathrm{b}}$ & $110(62-147)$ & NA & $143(72.6-288)$ & NA & $134.2(62-288)$ \\
\hline Albumin, $g / L^{b}$ & $23.8(7-33)$ & $27.4(21-40)$ & $28.0(13-40)$ & NA & $27.0(7-40)$ \\
\hline
\end{tabular}

$e G F R$ estimated glomerular filtration rate, $N A$ not applicable

${ }^{\text {a }}$ Patients $<18$ years of age

${ }^{\mathrm{b}}$ At baseline

Table 2 Available pharmacokinetic observations

\begin{tabular}{|c|c|c|c|c|c|c|c|c|}
\hline & \multicolumn{2}{|l|}{ Kenya } & \multicolumn{2}{|l|}{ Sudan } & \multicolumn{2}{|l|}{ Ethiopia } & \multicolumn{2}{|l|}{ India } \\
\hline & $\mathrm{PM}$ & $\mathrm{PM}+\mathrm{SSG}$ & $\mathrm{PM}$ & $\mathrm{PM}+\mathrm{SSG}$ & $\mathrm{PM}$ & $\mathrm{PM}+\mathrm{SSG}$ & PM & Total \\
\hline Subjects $(n)^{\mathrm{a}}$ & 4 & 12 & 10 & 6 & 22 & 20 & 454 & 528 \\
\hline Observations $(n)^{a}$ & 47 & 153 & 137 & 77 & 65 & 60 & 1333 & 1872 \\
\hline Observations BLQ $[n(\%)]$ & $14(30)$ & $32(21)$ & $32(23)$ & $23(30)$ & $6(9)$ & $4(7)$ & $277(21)$ & $388(21)$ \\
\hline Observations analysed $[n(\%)]^{\mathrm{b}}$ & $32(68)$ & $109(71)$ & $95(69)$ & $54(70)$ & $51(78)$ & $47(78)$ & $933(70)$ & $1321(71)$ \\
\hline Treatment duration & 21 & 17 & 21 & 17 & 21 & 17 & 21 & \\
\hline Sampling days & 1,21 & 1,17 & 1,21 & 1,17 & $7,14,21$ & $7,14,17$ & $1,8,15,21$ & \\
\hline
\end{tabular}

$P M$ paromomycin, $S S G$ sodium stibogluconate, $B L Q$ below the limit of quantification

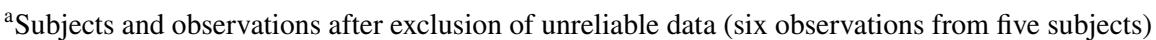

${ }^{\mathrm{b}}$ Observations after the exclusion of $T=0 \mathrm{~h}$ and $T=24 \mathrm{~h}$ observations and BLQ observations

for volume of distribution, only for the African populations (31.5\% [29.0\%]). Absorption from the intramuscular site of injection was fast, with a rate constant of $1.99 \mathrm{~h}^{-1}$ (6.52\%). Exposure in Eastern African patients was higher compared with Indian patients, which was best characterized by 1.17 -fold $(5.18 \%)$ higher bioavailability in Kenya and Sudan, and 2.46-fold (24.5\%) higher bioavailability in Ethiopia. To describe the higher variability among Ethiopian concentration-time profiles, between-subject variability was applied to F1 for the Ethiopian population (150\% [24.0\%]). Additionally, the deviating concentration-time profiles in Ethiopia were characterized by an approximately fourfold slower absorption rate constant of $0.446 \mathrm{~h}^{-1}(18.2 \%)$. On top of allometric scaling of clearance and volume of distribution for body weight, a linear relationship between age and clearance improved the model significantly ( $-57.6 \mathrm{OFV})$, resulting in a decline of clearance by $1.25 \%(10.6 \%)$ per year increase in age. For Eastern African patients only, a significant and clinically relevant exponential relationship between time and clearance was identified ( $-31.3 \mathrm{OFV})$, amounting to $\mathrm{a}-32.6 \%(15.2 \%)$ decrease in clearance between the start and end of treatment (day 21). A separate residual error for both studies improved the model significantly, with residual variabilities of $56.1 \%(2.96 \%)$ and $64.6 \%(4.54 \%)$ for the Indian and Eastern African data, respectively. No drug-drug interactions between paromomycin and SSG could be identified and paromomycin pharmacokinetics were dose proportional for the studied dose range. At the end of treatment, $\mathrm{AUC}_{\tau, \mathrm{SS}}$ for $15 \mathrm{mg} / \mathrm{kg} /$ day (median [interquartile range]) was higher in Kenya and Sudan (172.7 $\mu \mathrm{g} \cdot \mathrm{h} / \mathrm{mL}$ [145.9-214.3]) and in Ethiopia (230.1 $\mu \mathrm{g} \cdot \mathrm{h} / \mathrm{mL}$ [146.3-591.2]), compared with India $(97.26 \mu \mathrm{g} \cdot \mathrm{h} / \mathrm{mL}$ [80.83-123.4]) (Table 4).

\subsection{Model Evaluation}

The final parameters of the population pharmacokinetic model were adequately estimated (Table 3 ), with acceptable parameter precisions based on SIR $(<30 \%$ RSE), shrinkages of between-subject variabilities $(<40 \%)$, correlations between parameters $(\leq 0.6)$, and a low condition number (13.59). Population and individual model predictions were adequately describing the observations, with no major trends 


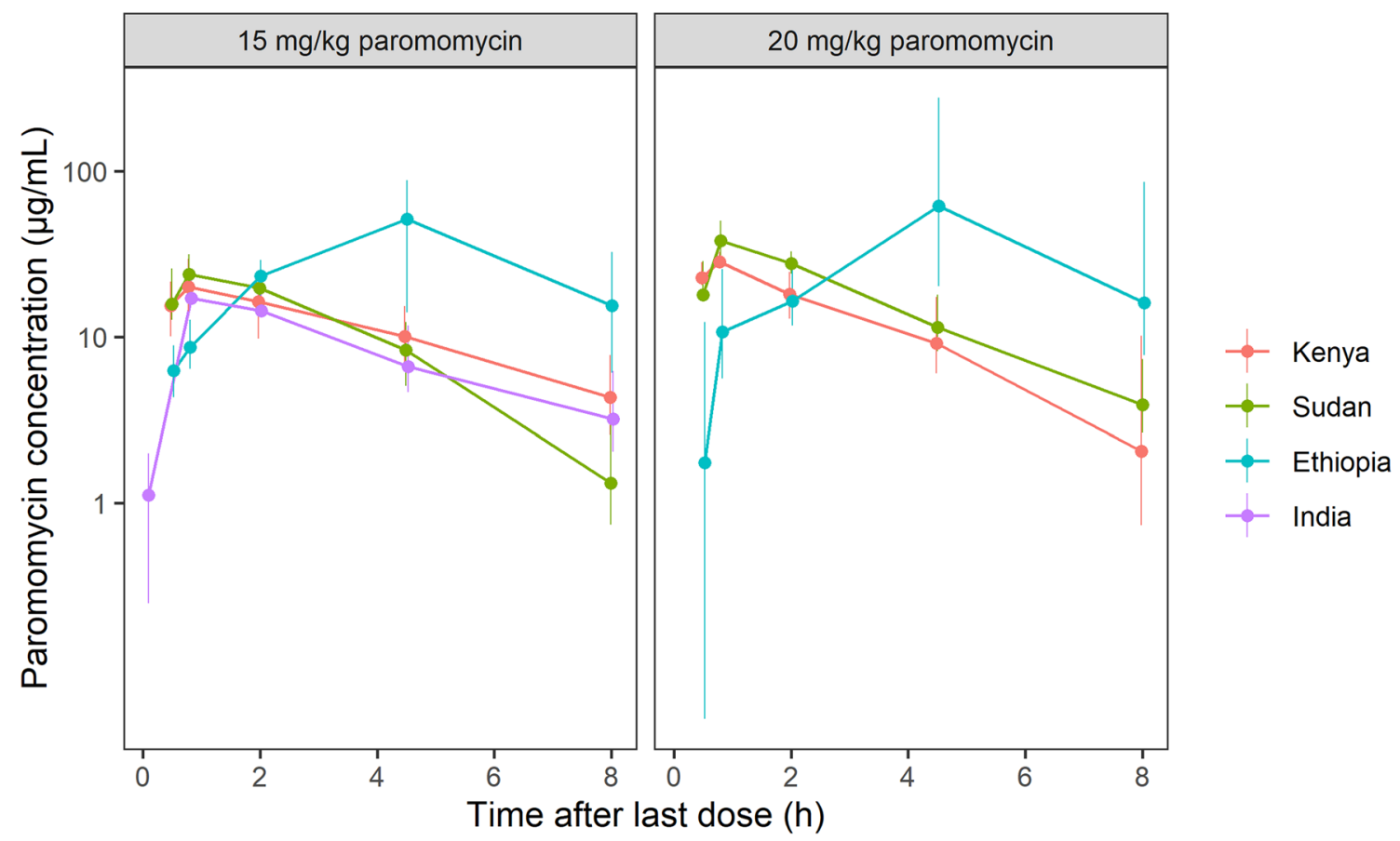

Fig. 1 Paromomycin plasma concentrations (median and interquartile range) of all sampling days included in the pharmacokinetic analysis, stratified by country and paromomycin dose

Table 3 Parameter estimates of the final population pharmacokinetic model

\begin{tabular}{|c|c|c|c|}
\hline & Estimate & $95 \% \mathrm{CI}^{\mathrm{a}}$ & Shrinkage $(\%)$ \\
\hline \multicolumn{4}{|l|}{ Population parameters } \\
\hline $\mathrm{Cl}_{\text {pop }}(\mathrm{L} / \mathrm{h})$ & 4.38 & $4.17-4.59$ & \\
\hline$V_{\mathrm{d} \text {,pop }}(\mathrm{L})$ & 15.6 & $15.0-16.2$ & \\
\hline$k_{\mathrm{a}, \mathrm{pop}}\left(\mathrm{h}^{-1}\right)$ & 1.99 & $1.76-2.27$ & \\
\hline $\mathrm{F} 1_{\text {pop }}$ & 1.00 (fixed) & & \\
\hline $\operatorname{COV}_{\mathrm{Cl}, \text { time }}\left(\mathrm{h}^{-1}\right)$ & -0.000782 & -0.00101 to -0.000561 & \\
\hline $\mathrm{COV}_{\mathrm{Cl}, \text { age }}\left(\right.$ year $\left.^{-1}\right)$ & -0.0125 & -0.0151 to -0.0100 & \\
\hline$k_{\mathrm{a}, \text { Ethiopia }}\left(\right.$ fold change $\left.k_{a, \text { pop }}\right)$ & 0.224 & $0.151-0.305$ & \\
\hline $\mathrm{F} 1_{\text {Ethiopia }}\left(\right.$ fold change $\left.\mathrm{F} 1_{\text {pop }}\right)$ & 2.46 & $1.59-4.01$ & \\
\hline $\mathrm{F} 1_{\text {Kenya,Sudan }}$ (fold change $\mathrm{F} 1_{\text {pop }}$ ) & 1.17 & $1.06-1.30$ & \\
\hline \multicolumn{4}{|l|}{ Between-subject variability } \\
\hline $\mathrm{Cl}(\mathrm{CV} \%)$ & 33.2 & $30.1-36.5$ & 29 \\
\hline$V_{\mathrm{d}, \text { Eastern-Africa }}(\mathrm{CV} \%)$ & 31.5 & $23.3-41.0$ & 40 \\
\hline $\mathrm{F} 1_{\text {Ethiopia }}(\mathrm{CV} \%)$ & 150 & $120.3-189.1$ & 8 \\
\hline \multicolumn{4}{|l|}{ Residual variability } \\
\hline Proportional error India (CV\%) & 56.1 & $54.6-57.9$ & 10 \\
\hline Proportional error Eastern Africa (CV\%) & 64.6 & $62.1-67.7$ & 10 \\
\hline
\end{tabular}

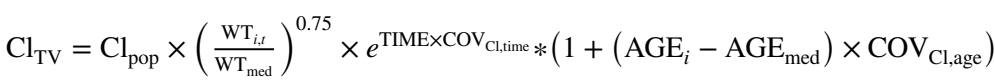

$V_{d, \mathrm{TV}}=V_{d, \mathrm{pop}} \times\left(\frac{\mathrm{WT}_{i, t}}{\mathrm{WT}_{\mathrm{med}}}\right)^{1.00}$

$A G E_{i}$ individual age (years), $A G E_{\text {med }}$ median population age (20 years), $C I$ confidence interval, $C l_{\text {pop }}$ population apparent oral clearance, $C l_{T V}$ typical value apparent oral clearance, $C O V_{C l, a g e}$ scaling factor covariate age on clearance, $C O V_{C l, t i m e}$ scaling factor covariate time on clearance, $C V \%$ percentage coefficient of variation, $F 1_{\text {Ethiopia }}$ proportional change of $\mathrm{F} 1$ in Ethiopia, $F 1_{\text {Kenya,Sudan }}$ proportional change of $\mathrm{F} 1$ in Kenya and Sudan, $F 1_{\text {pop }}$ population bioavailability, $\mathrm{k}_{\mathrm{a} \text {,Ethiopia }}$ proportional change of $\mathrm{k}_{\mathrm{a}}$ in Ethiopia, $k_{a, p o p}$ population absorption rate constant, SIR sampling-importance resampling, TIME time after start of treatment, $V_{d \text {,Eastern-Africa }}$ central volume of distribution in Eastern African patients, $V_{d, p o p}$ population central volume of distribution, $V_{d, T V}$ typical value central volume of distribution, $W T_{i, t}$ individual body weight $(\mathrm{kg})$ at time $t, W T_{\text {med }}$ median population body weight $(39 \mathrm{~kg})$

a Obtained by SIR 
visible in the GoF plots (Fig. 2). Simulation-based diagnostics indicated an overall good predictive performance of the model, although the plasma concentrations in the absorption phase in the Ethiopian population were slightly overpredicted, illustrated by the VPC (Fig. 3). The different variability in plasma concentrations between countries was well-described by the model (Fig. 3).

\section{Discussion}

The population pharmacokinetic model adequately described paromomycin pharmacokinetics in VL patients from different Eastern African countries and India. A decline in clearance during treatment was observed, as well as a decline in clearance with age. No drug-drug interactions were observed with SSG, and paromomycin pharmacokinetics were dose proportional for the studied dose range.

The shape and variability of the concentration-time profiles in Ethiopian patients differed greatly from the other
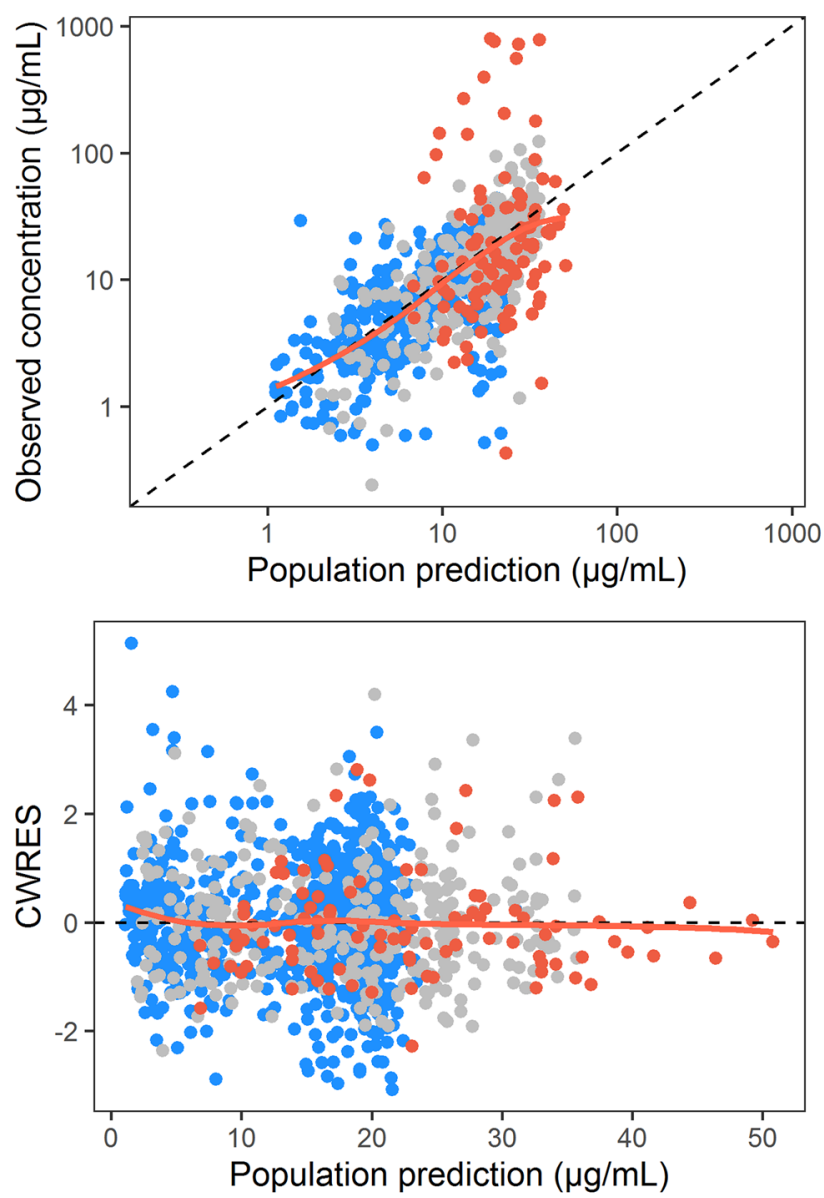

Fig. 2 Goodness-of-fit plots for the final population pharmacokinetic model, coloured by country. Model-predicted population and individual paromomycin concentrations versus observed concentrations,
Eastern African and Indian patient populations. The differences were best characterized by a slower absorption rate (fourfold slower) and a higher typical intramuscular bioavailability (2.46-fold), but with a very high betweensubject variability of this bioavailability $(150 \%)$. The sparse sampling scheme used in Ethiopia cannot be responsible for the observed pharmacokinetic differences, as the population pharmacokinetic approach accounts for sparse and heterogeneous data. The fast absorption rate of $1.99 \mathrm{~h}^{-1}$ observed in Kenya, Sudan and India is more in line with the absorption rate of $2.65 \mathrm{~h}^{-1}$ shown earlier in seven healthy volunteers receiving the same paromomycin dose regimen [8]. Variable absorption rates and bioavailability have been observed for other aminoglycosides such as amikacin and gentamicin $[19,20]$, although not as extreme as we observed in this study. Possible explanations were considered. The same paromomycin product and batch was used in all sites in Eastern Africa, but differences in, for example, storage conditions (altering product stability and/or solubility), different conditions during sample transport and analysis (altering
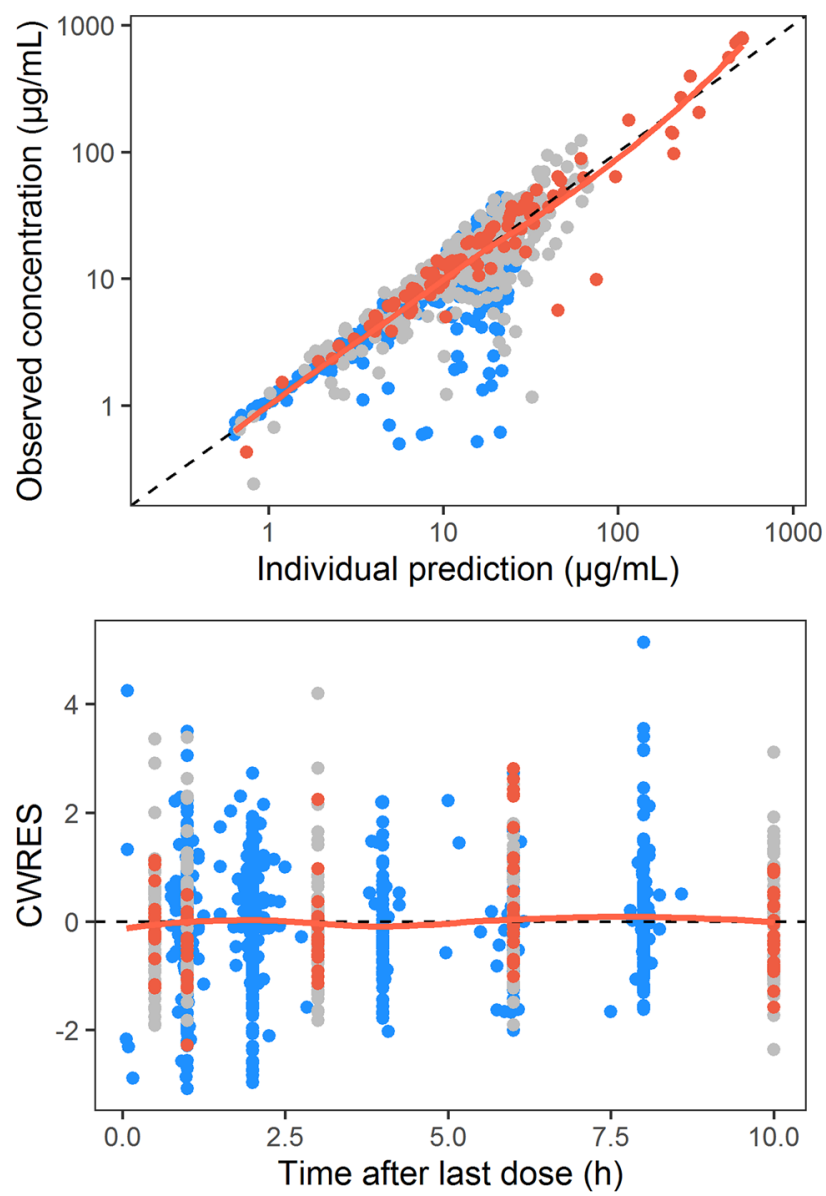

and CWRES versus time after last dose and population predictions, for Kenya and Sudan (grey), Ethiopia (red) and India (blue). CWRES conditional weighted residuals 


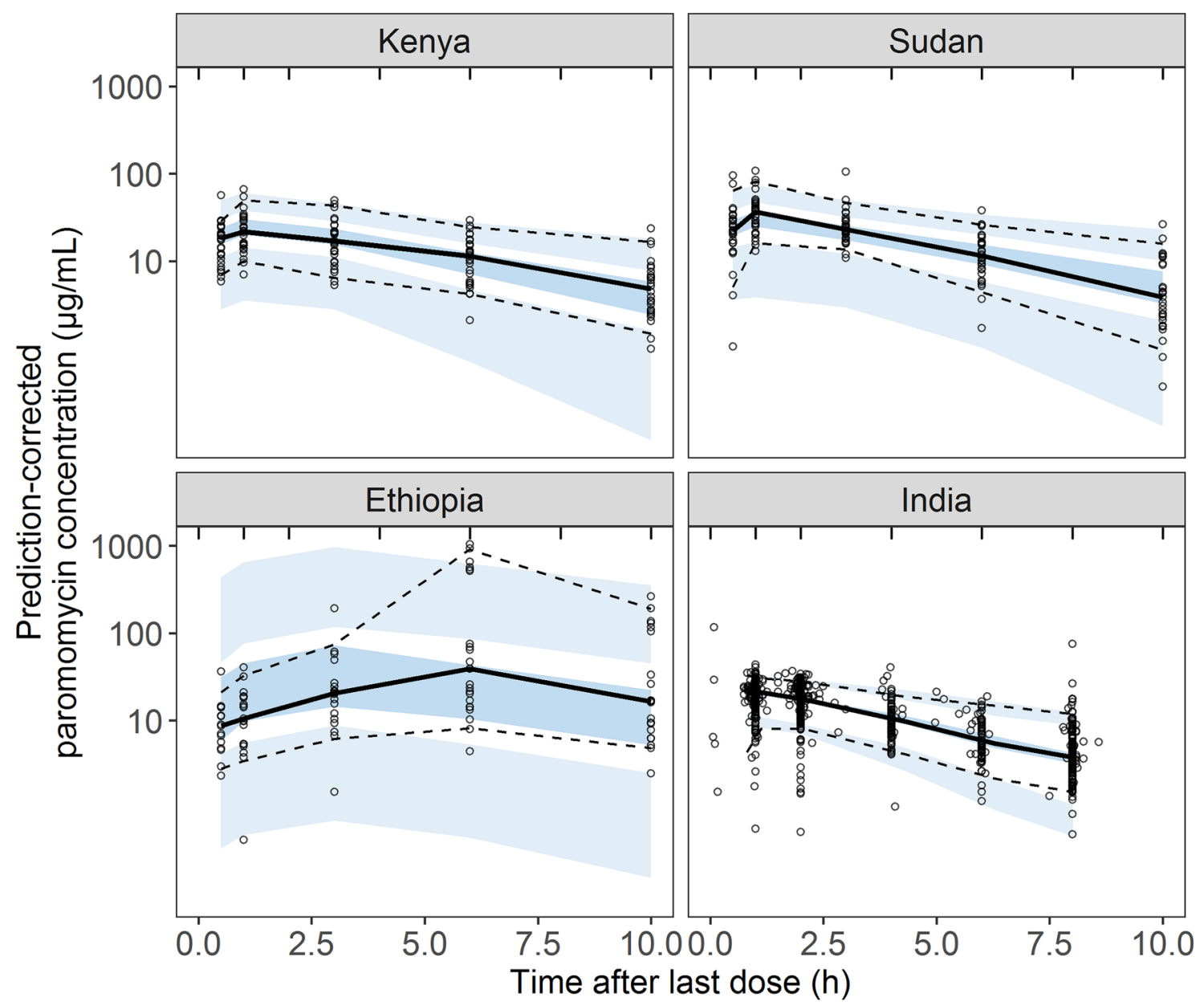

Fig. 3 Prediction-corrected visual predictive check of the final paromomycin pharmacokinetic model stratified by country. Solid and dashed lines represent the 50th, 5th and 95th percentiles of the

Table 4 Paromomycin exposure by dose and country

\begin{tabular}{llc}
\hline Dose & Country & $\mathrm{AUC}_{\tau, \mathrm{SS}}[\mu \mathrm{g} \cdot \mathrm{h} / \mathrm{mL}]^{\mathrm{a}}$ \\
\hline $15 \mathrm{mg} / \mathrm{kg}$ & Kenya & $188.4(151.4-233.5)$ \\
& Sudan & $149.4(137.6-170.8)$ \\
& Ethiopia & $230.1(146.3-591.2)$ \\
$20 \mathrm{mg} / \mathrm{kg}$ & India & $97.26(80.83-123.4)$ \\
& Kenya & $215.0(142.8-337.0)$ \\
& Sudan & $287.8(244.5-341.6)$ \\
& Ethiopia & $235.9(117.7-391.2)$ \\
\hline
\end{tabular}

$A U C_{\tau, S S}$ area under the plasma concentration-time curve for $24 \mathrm{~h}$ at steady-state, determined on the last day concentrations were measured, $I Q R$ interquartile range

${ }^{\mathrm{a}}$ Median (IQR)

analyte stability in the samples), or exact site of injections (e.g. subdermal instead of intramuscular) between study sites and/or trials cannot be fully excluded. Notable in this observed values, whereas the dark and light blue areas indicate the $90 \%$ confidence intervals of the simulated 50th, 5th and 95th percentiles, based on 1000 simulations

respect is that Ethiopian patients had been included at two distinct sites in North and South Ethiopia, but no differences in pharmacokinetics could be identified between those two sites (data not shown). Alterations in body composition and physiological changes are associated with malnutrition and/ or clinical manifestations of VL [21, 22], which might also impact pharmacokinetics. However, we could not identify any correlations between age or factors related to malnutrition or disease severity (body weight, serum albumin) and exposure, clearance or drug absorption.

A priori, we expected a correlation between serum creatinine or GFR and paromomycin clearance, as paromomycin is mainly cleared renally. However, the inclusion of these covariates did not significantly improve the model fit, probably because all Eastern African patients in this study had relatively normal serum creatinine levels (range 44.2-134 $\mu \mathrm{mol} / \mathrm{L})$. Second, to find a mechanistic explanation for the decline in clearance, the effect of time-varying serum albumin was evaluated on volume of distribution and clearance. 
Patients had hypoalbuminaemia at the start of treatment (range 7-40 g/L), which increased during and after treatment (range $20-57 \mathrm{~g} / \mathrm{L}$ at the 2-month follow-up after the end of treatment). In treatment with other aminoglycosides, decreased albumin has been correlated with increased volume of distribution [23-26], which in turn can cause a decrease in maximum concentration $\left(\mathrm{C}_{\max }\right)[24,26]$ and possibly a lower renal clearance. The increase in serum albumin over time corresponds to the decrease in clearance over time. However, we did not find any significant effect of timevarying albumin levels on any pharmacokinetic parameters.

In this pooled pharmacokinetic analysis, data were retrieved from different trials with different treatment regimens (different paromomycin milligram per kilogram dose, with and without a companion drug) and sampling schemes were different. To optimally analyse the heterogenic data, a population approach was used, allowing to adequately characterize paromomycin pharmacokinetics, to link geographical differences to certain pharmacokinetic parameters, and to describe part of the variability by explanatory covariates. Moreover, the population approach allows adequate analysis of different sampling schemes, e.g. the sparse sampling scheme used in Ethiopian sites. However, the retrospective design of the study brings limitations. Serum creatinine and albumin data were missing in the Indian population, and therefore these covariates could only be evaluated in a subset of patients. Second, $27.2 \%$ of data of paromomycin plasma levels at dosing time points (TAD $=0$ and TAD $=24 \mathrm{~h}$ ) were excluded because the data were highly variable and physiologically implausible. However, exclusion of these data did not lead to any substantial changes in identification and parameter estimates of the structural pharmacokinetic model, implying a negligible effect on subsequent model development.

Surprisingly, the substantial geographical differences in exposure that were identified in this study were not in line with the observed geographical variability in efficacy. Efficacy rates after a lower paromomycin dose $(15 \mathrm{mg} / \mathrm{kg}$ paromomycin for 21 days) [3] were in line with exposure differences among Eastern African countries in the current study, which was lowest in Sudan (14.3\% and 46.7\%) and highest in Southern Ethiopia (96.6\%) [3]. Contrarily, exposure in Sudanese patients was higher compared with Kenyan and Ethiopian patients when receiving $20 \mathrm{mg} /$ $\mathrm{kg}$ paromomycin. For this dose level, geographical efficacy differences within Eastern Africa were not observed, although the current clinical trial was not powered to perform a by-site analysis [5]. Moreover, high efficacy in Indian patients (94.6\%) [2] was observed $(15 \mathrm{mg} / \mathrm{kg}$ for 21 days), while exposure was significantly lower compared with all Eastern African countries. Therefore, the reported differences in efficacy were most probably not related to the pharmacokinetics. While the pharmacokinetic substudies included, for example, less children than the total clinical trial populations on which the efficacy figures were based, there are no indications that there are significant efficacy differences between children and adults at the evaluated dose levels $[2,5]$. The lower efficacy in Sudan compared with India might be related to the virulence of the Leishmania parasite or to immunological and pathophysiological differences between populations, while, to date, parasite susceptibility differences have not been documented in vitro. Moreover, this trend of lowered efficacy in Eastern Africa is not only observed for paromomycin treatment but also for other VL treatment regimens [27].

\section{Conclusion}

This pooled population pharmacokinetic model provides detailed insight into the geographical pharmacokinetic differences among Eastern African countries and India; however, the resulting differences in paromomycin pharmacokinetics most probably do not explain the geographical differences in efficacy of paromomycin monotherapy in the treatment of VL patients. Potential explanations for these efficacy differences might be host-related, e.g. immunological or genetic differences, or parasite-related e.g. parasite virulence differences. Moreover, this analysis demonstrates the relevance of pharmacokinetic analysis in these populations, as remarkable differences in exposure were observed between geographic populations, despite the use of a body weight-guided dosing regimen.

Supplementary Information The online version contains supplementary material available at https://doi.org/10.1007/s40262-021-01036-8.

Acknowledgements The authors would like to thank the Institute for One World Health (iOWH), now referred to as PATH, and all members of the field teams, including nurses and laboratory technicians in all study sites, clinical monitors and Data Safety Monitoring Boards for their contribution to the original studies. The authors sincerely thank the VL patients and the parents and guardians of the paediatric patients for their willingness to be enrolled in this study and their cooperation.

\section{Declarations}

Conflicts of interest Luka Verrest, Monique Wasunna, Gilbert Kokwaro, Rashid Aman, Ahmed M. Musa, Eltahir A.G. Khalil, Mahmoud Mudawi, Brima M. Younis, Asrat Hailu, Zewdu Hurissa, Workagegnehu Hailu, Samson Tesfaye, Eyasu Makonnen, Yalemtsehay Mekonnen, Alwin D.R. Huitema, Jos H. Beijnen, Smita A. Kshirsagar, Jaya Chakravarty, Madhukar Rai, Shyam Sundar, Fabiana Alves and Thomas P.C. Dorlo have no conflicts of interest to declare related to this study.

Funding The Eastern African clinical study was funded by Médecins Sans Frontières (MSF) and the Drugs for Neglected Diseases initiative (DNDi). The authors would like to thank the following donors for their support: Department for International Development (DFID), 
UK; Médecins Sans Frontières/Doctors without Borders, International; Ministry of Foreign and European Affairs (MAEE), France; Region of Tuscany, Italy; République and Canton de Geneva, Switzerland; Medicor Foundation, Liechtenstein; Fondation Pro Victimis, Switzerland; Fondation André and Cyprien, Switzerland; Spanish Agency for International Development Cooperation (AECID), Spain; Swiss Agency for Development and Cooperation (SDC), Switzerland; and private foundations and individual donors. The Indian clinical study was supported by the Bill and Melinda Gates Foundation, the Institute for One World Health, the Special Program for Research and Training in Tropical Diseases (TDR) of the United Nations Development Program, the World Bank, and the WHO. The current pharmacokinetic analysis was part of the EDCTP2 programme supported by the European Union (grant number RIA2016S1635-AfriKADIA). TPCD received funding from ZonMw/Dutch Research Council (NWO) [project number 91617140].

Availability of data and material The NONMEM control stream can be found in ESM 3. For availability of the datasets analysed during the current study, please contact the corresponding author.

Ethical approval and informed consent All procedures were conducted in accordance with the Declaration of Helsinki (2002 version) relating to the conduct of research on human subjects, and followed the International Committee on Harmonization guidelines for the conduct of clinical trials. The relevant Ethics Committees from each country approved the clinical studies. Patients or their legal guardians (if they were minors) provided signed informed consent prior to being randomized to the different treatment arms. In India, patients were randomly assigned to the pharmacokinetic substudy, while in Eastern Africa, patients were asked to participate in the pharmacokinetic substudy using a separate informed consent.

Author contributions All authors participated in the collection of the study data, interpretation of the study results, and drafting, critical revision and approval of the final version of the manuscript. $\mathrm{LV}, \mathrm{AH}$, FA, and TD were involved in the design of the analysis. LV and TD were responsible for data analysis and a draft of the first version of the manuscript.

\section{Consent For Publication Not applicable.}

Open Access This article is licensed under a Creative Commons Attribution-NonCommercial 4.0 International License, which permits any non-commercial use, sharing, adaptation, distribution and reproduction in any medium or format, as long as you give appropriate credit to the original author(s) and the source, provide a link to the Creative Commons licence, and indicate if changes were made. The images or other third party material in this article are included in the article's Creative Commons licence, unless indicated otherwise in a credit line to the material. If material is not included in the article's Creative Commons licence and your intended use is not permitted by statutory regulation or exceeds the permitted use, you will need to obtain permission directly from the copyright holder. To view a copy of this licence, visit http://creativecommons.org/licenses/by-nc/4.0/.

\section{References}

1. World Health Organization. Recommended treatment regimens for visceral leishmaniasis. https://www.who.int/leishmaniasis/resea rch/978924129496_pp67_71.pdf?ua=1. Accessed 18 Oct 2020.
2. Sundar S, Jha TK, Thakur CP, Sinha PK, Bhattacharya SK. Injectable paromomycin for visceral leishmaniasis in India. N Engl J Med. 2007;356:2571-81.

3. Hailu A, Musa A, Wasunna M, Balasegaram M, Yifru S, Mengistu $\mathrm{G}$, et al. Geographical variation in the response of visceral leishmaniasis to paromomycin in East Africa: A multicentre, openlabel, randomized trial. PLoS Negl Trop Dis. 2010;4:e709.

4. Musa AM, Younis B, Fadlalla A, Royce C, Balasegaram M, Wasunna M, et al. Paromomycin for the treatment of visceral leishmaniasis in Sudan: A randomized, open-label, dose-finding study. PLoS Negl Trop Dis. 2010;4:4-10.

5. Musa A, Khalil E, Hailu A, Olobo J, Balasegaram M, Omollo R, et al. Sodium stibogluconate (SSG) \& paromomycin combination compared to SSG for visceral leishmaniasis in East Africa: A randomised controlled trial. PLoS Negl Trop Dis. 2012;6:e1674.

6. Verrest L, Dorlo TPC. Lack of clinical pharmacokinetic studies to optimize the treatment of neglected tropical diseases: a systematic Review. Clin Pharmacokinet. 2017;56:583-606.

7. Kip AE, Schellens JHM, Beijnen JH, Dorlo TPC. Clinical pharmacokinetics of systemically administered antileishmanial drugs. Clin Pharmacokinet. 2018;57:151-76.

8. Kanyok TP, Killian AD, Rodvold KA, Danziger LH. Pharmacokinetics of intramuscularly administered aminosidine in healthy subjects. Antimicrob Agents Chemother. 1997;41:982-6.

9. Seyffart G. Drug dosage in renal insufficiency. Springser Science Business Media; 1991

10. Institute for One World Health. Application for inclusion of paromomycin in the WHO Model List of Essential Medicines. 2006. http://archives.who.int/eml/expcom/expcom15/applications/ newmed/paromomycin/paromomycin.pdf. Accessed 18 Oct 2020.

11. Beal SL. Ways to fit a PK model with some data below the quantification limit. J Pharmacokinet Pharmacodyn. 2001;28:481-504.

12. Anderson BJ, Holford NHG. Mechanism-based concepts of size and maturity in pharmacokinetics. Annu Rev Pharmacol Toxicol. 2008;48:303-32.

13. Stevens LA, Coresh J, Feldman HI, Greene T, Lash JP, Nelson $\mathrm{RG}$, et al. Evaluation of the modification of diet in renal disease study equation in a large diverse population. J Am Soc Nephrol. 2007; 18:2749-57.

14. Du Bois D, Du Bois EF. A formula to estimate the approximate surface area if height and weight be known. 1916. Nutrition. 1989;5:303.

15. Varma N, Naseem S. Hematologic changes in visceral Leishmaniasis/Kala Azar. Indian J Hematol Blood Transfus. 2010;26:78-82.

16. Karlsson MO, Savic RM. Diagnosing model diagnostics. Clin Pharmacol Ther. 2007;82:17-20.

17. Bergstrand M, Hooker AC, Wallin JE, Karlsson MO. Prediction-corrected visual predictive checks for diagnosing nonlinear mixed-effects models. AAPS J. 2011;13:143-51.

18. Dosne AG, Bergstrand M, Karlsson MO. An automated sampling importance resampling procedure for estimating parameter uncertainty. J Pharmacokinet Pharmacodyn. 2017;44:509-20.

19. Thomson AH, Kokwaro GO, Muchohi SN, English M, Mohammed S, Edwards G. Population pharmacokinetics of intramuscular gentamicin administered to young infants with suspected severe sepsis in Kenya. Br J Clin Pharmacol. 2003;56:25-31.

20. Pfeffer M, van Harken DR. Effect of dosing volume on intramuscular absorption rate of aminoglycosides. J Pharm Sci. 1981;70(4):449-52.

21. Feleke BE. Nutritional status of visceral leishmaniasis patients: a comparative cross-sectional study. Clin Nutr ESPEN. 2019;33:139-42.

22. Mengesha B, Endris M, Takele Y, Mekonnen K, Tadesse T, Feleke $\mathrm{A}$, et al. Prevalence of malnutrition and associated risk factors 
among adult visceral leishmaniasis patients in Northwest Ethiopia: a cross sectional study. BMC Res Notes. 2014;7:1-6.

23. Davis RL, Lehmann D, Stidley CA, Neidhart J. Amikacin pharmacokinetics in patients receiving high-dose cancer chemotherapy. Antimicrob Agents Chemother. 1991;35:944-7.

24. Etzel JV, Nafziger AN, Bertino JS. Variation in the pharmacokinetics of gentamicin and tobramycin in patients with pleural effusions and hypoalbuminemia. Antimicrob Agents Chemother. 1992;36:679-81.

25. Romano S, de Gatta FMM, Calvo MV, Caballero D, DominquezGil A, Lanao JM. Population pharmacokinetics of amikacin in patients with haematological malignancies. J Antimicrob Chemother. 1999;44:235-42.

26. Hodiamont CJ, Juffermans NP, Bouman CSC, De JMD, Mathôt RAA, Van HRM. Determinants of gentamicin concentrations in critically ill patients: a population pharmacokinetic analysis. Int J Antimicrob Agents. 2017;49:204-11.

27. Alves F, Bilbe G, Blesson S, Goyal V, Monnerat S, Mowbray C, et al. Recent development of visceral leishmaniasis treatments: successes, pitfalls, and perspectives. Clin Microbiol Rev. 2018;31:1-30

\section{Authors and Affiliations}

Luka Verrest $^{1} \cdot$ Monique Wasunna $^{2} \cdot$ Gilbert Kokwaro $^{3,4} \cdot$ Rashid Aman $^{4} \cdot$ Ahmed M. Musa $^{5}$ - Eltahir A. G. Khalii ${ }^{5}$. Mahmoud Mudawi $^{5,6} \cdot$ Brima M. Younis $^{5}$. Asrat Hailu ${ }^{7}$. Zewdu Hurissa ${ }^{8}$. Workagegnehu Hailu ${ }^{9}$. Samson Tesfaye ${ }^{7}$. Eyasu Makonnen ${ }^{7}$ Yalemtsehay Mekonnen ${ }^{10}$. Alwin D. R. Huitema ${ }^{1,11,12} \cdot$ Jos H. Beijnen $^{1} \cdot$ Smita A. Kshirsagar $^{13}$. Jaya Chakravarty ${ }^{14} \cdot$ Madhukar Rai $^{14}$. Shyam Sundar ${ }^{14}$. Fabiana Alves ${ }^{15}$. Thomas P. C. Dorlo ${ }^{1}$

1 Department of Pharmacy and Pharmacology, The Netherlands Cancer Institute/Antoni van Leeuwenhoek Hospital, PO Box 90440, 1006 BK Amsterdam, The Netherlands

2 Drugs for Neglected Diseases initiative (DNDi) Africa, Nairobi, Kenya

3 KEMRI Wellcome Trust Programme, Nairobi, Kenya

4 African Centre for Clinical Trials, Nairobi, Kenya

5 Institute of Endemic Diseases, University of Khartoum, Khartoum, Sudan

6 Department of Pharmacology and Toxicology, Faculty of Pharmacy, Northern Border University, Arar, Saudi Arabia

7 College of Health Sciences, Addis Ababa University, Addis Ababa, Ethiopia

8 College of Health Sciences, Arsi University, Asella, Ethiopia
9 College of Medicine and Health Sciences, University of Gondar, Gondar, Ethiopia

10 College of Natural and Computational Sciences, Addis Ababa University, Addis Ababa, Ethiopia

11 Department of Clinical Pharmacy, University Medical Center Utrecht, Utrecht University, Utrecht, The Netherlands

12 Department of Pharmacology, Princess Máxima Center for Pediatric Oncology, Utrecht, The Netherlands

13 Department of Medicine, Stanford University Medical Center, Stanford, CA, USA

14 Institute of Medical Sciences, Banaras Hindu University, Varanasi, Uttar Pradesh, India

15 Drugs for Neglected Diseases initiative (DNDi), Geneva, Switzerland 